\title{
Respiratory muscle training in patients with stroke
}

\author{
Ana Lista-Paz $\mathrm{PhD}^{1}$; Rodrigo Torres-Castro $\mathrm{MSc}^{2}$; Guilherme Fregonezi $\mathrm{PhD}^{3}$; \\ Luz González Doniz $\mathrm{PhD}^{1}$
}

${ }^{1}$ Faculty of Physiotherapy University of A Coruña A Coruña, Spain. ${ }^{2}$ Department of Physical Therapy Faculty of Medicine University of Chile Santiago, Chile. ${ }^{3}$ PneumoCardioVascular Lab Hospital Universitário Onofre Lopes Empresa Brasileira de Serviços Hospitalares (EBSERH) \& Departamento de Fisioterapia Universidade Federal do Rio Grande do Norte Natal, Brazil.

To the Editor:

We have read with great interest the article written by Yoo and Pyun ${ }^{1}$ entitled "Efficacy of Bedside Respiratory Muscle Training in Patients With Stroke." 1 The authors conducted a randomized clinical trial in 40 patients with stroke within less than 3 months of evolution (20 in the intervention group and 20 in the control group) with those who performed a protocol of 3 weeks of muscular respiratory training based on exercises of respiratory control, flow-oriented incentive spirometry, and Acapella vibratory positive expiratory pressure device.

It is known that patients with stroke have respiratory muscle weakness. ${ }^{2}$ This weakness that appears in the acute stage of the disease and remains in the chronic period decreases the efficiency of the respiratory system by which it produces a decrease in lung volumes and alteration of the mechanism of cough, increasing morbidity and mortality due to respiratory diseases. ${ }^{3,4}$ In addition, respiratory muscle weakness in the chronic phase of stroke leads to important functional and cardiorespiratory limitations. ${ }^{5}$ The sum of this weakness, together with alterations in respiratory mechanics, the spasticity and contractures of trunk muscles, and a reduced mobility of the affected diaphragmatic dome, could lead to a restrictive ventilatory pattern. ${ }^{4,6}$ Therefore, it is necessary to continue investigating the effects of stroke on respiratory muscles and the use of training of respiratory muscles as a rehabilitation technique.

The authors describe a training protocol for inspiratory and expiratory muscles. However, it is important to highlight that respiratory muscle training, as well as the training of any other muscle, must comply with certain principles: overload, specificity, and reversibility. Although the duration and frequency of training continue to be the subject of discussion, the key to the training prescription is the correct dosage of its intensity or load, as well as the total time or volume of training. ${ }^{7}$ 
The most commonly used test in the clinical field for the evaluation of the respiratory muscle strength is the measurement of the maximum respiratory pressures in the mouth: the maximum inspiratory pressure and the maximum expiratory pressure. From these values, reference values have been established for a healthy population and countless publications provide values for different diseases. ${ }^{8}$

The authors did not report the value of maximum inspiratory pressure and maximum expiratory pressure, so the degree of muscle weakness or the training load cannot be determined. With the data provided, only the peak expiratory flow is available as an indirect measure of the state of the expiratory muscles and whose value is approximately $200 \mathrm{l} / \mathrm{min}$, very close to the threshold of an ineffective cough. ${ }^{9}$

Having the maximum respiratory pressure value is essential, not only to dose the intensity of initial training but also to adjust the workload during the duration of the muscle training program and, once completed, to set the measures of maintenance of strength through the continuity of home-based training, considering that the devices used to train the muscles are portable.

Another important point is the protocol used. The authors cite in methods a recent systematic review in which training protocols are described, emphasizing devices that have shown clinical and statistically significant improvement. ${ }^{2}$ This review shows five clinical trials in which threshold devices were used, which do not depend on flow and in which a training load can be objectively established. That is, from the maximum inspiratory pressure, you can train a percentage of it, which is usually established in a range between $30 \%$ and $80 \%$. The literature has consistently demonstrated, in different types of pathologies, that threshold devices with loads greater than $30 \%$ produce improvements in the strength and resistance of respiratory muscles. ${ }^{10-12}$ However, Yoo and Pyun, ${ }^{1}$ for inspiratory muscle training, used a flow-oriented incentive spirometer, whose main indication is the prevention of postsurgical complications, such as atelectasis, as well as in the prehabilitation of surgical patients, by contributing to increasing the volume and expansion of the pulmonary parenchyma. This device lacks a regulation system by pressure, which does not allow to establish the objective load of training. There are even investigations that report that the maximum pressure generated by this type of device does not exceed $10 \mathrm{cmH} 2 \mathrm{O} .{ }^{13}$ Regarding the training of the expiratory muscles, the authors used the Acapella device that generates positive expiratory pressure with endogenous vibration with the fundamental objective of improving the rheology of the secretions and favoring their drainage. ${ }^{14}$ This device has been shown to generate very low pressures that depend on expiratory flow ranging from 4 to $21 \mathrm{cmH}_{2} \mathrm{O}{ }^{15}$

We believe that the work of Yoo and Pyung ${ }^{1}$ is a very important step that shows significant results in the variables of pulmonary function before and after intervention with the use of two instruments whose main objectives are the increase of lung volumes (flow-oriented incentive spirometer) and secretion drainage (Acapella). However, it cannot be asserted that the result is due to the training of the respiratory muscles as the authors conclude. It has already been reported that patients with stroke have a significant volume decrease in the hemiplegic side that can reach up to $25 \%$ and that this decrease is normalized with the use of volume incentives as used by the authors. ${ }^{6}$

We are deeply convinced that the evaluation of the respiratory system after a stroke is fundamental in this population and that the incorporation of respiratory therapeutic interventions associated with neurorehabilitation programs should be part of the process of integral recovery of people who have experienced a stroke, to improve their quality of life and survival. It is important to continue research into the role played by the respiratory muscles in the cardiorespiratory conditioning process in stroke, but to draw reliable conclusions, precise evaluation procedures, key parameters, appropriate protocols, and the indicated training instruments should be selected. 


\section{REFERENCES}

1. Yoo HJ, Pyun SB: Efficacy of bedside respiratory muscle training in patients with stroke: a randomized controlled trial. Am J Phys Med Rehabil 2018;97:691-7

2. Menezes KK, Nascimento LR, Ada L, et al: Respiratory muscle training increases respiratory muscle strength and reduces respiratory complications after stroke: a systematic review. J Physiother 2016;62:138-44

3. Cabral EEA, Resqueti VR, Lima INDF, et al: Effects of positive expiratory pressure on chest wall volumes in subjects with stroke compared to healthy controls: a case-control study. Braz J Phys Ther 2017;21:416-24

4. Menezes KK, Nascimento LR, Avelino PR, et al: Efficacy of interventions to improve respiratory function after stroke. Respir Care 2018;63:920-33

5. Lista Paz A, González Doniz L, Ortigueira García S, et al: Respiratory muscle strength in chronic stroke survivors and its relation with the 6-minute walk test. Arch Phys Med Rehabil 2016;97:266-72

6. Lima IN, Fregonezi GA, Melo R, et al: Acute effects of volume-oriented incentive spirometry on chest wall volumes in patients after a stroke. Respir Care 2014;59:1101-7

7. Astrand P-O, Rodahl K: Textbook of Work Physiology: Physiological Bases of Exercise. 3rd ed. London, UK, McGraw-Hill, 1986

8. American Thoracic Society/European Thoracic Society: ATS/ERS statement on respiratory muscle testing. Am J Respir Crit Care Med 2002;166:518-624

9. Torres-Castro R, Monge G, Vera R, et al: Therapeutic strategies to increase the effectiveness of cough. Rev Med Chile 2014;142:238-45

10. Beaumont M, Forget $\mathrm{P}$, Couturaud F, et al: Effects of inspiratory muscle training in COPD patients: a systematic review and meta-analysis. Clin Respir J 2018;12:2178-88

11. Human A, Corten L, Jelsma J, et al: Inspiratory muscle training for children and adolescents with neuromuscular diseases: a systematic review. Neuromuscul Disord 2017;27:503-17

12. Karanfil EOT, Møller AM: Preoperative inspiratory muscle training prevents pulmonary complications after cardiac surgery — a systematic review. Dan Med J 2018;65(3). piiA5450

13. Weindler J, Kiefer RT: The efficacy of postoperative incentive spirometry is influenced by the device-specific imposed work of breathing. Chest 2001;119:1858-64

14. Patterson JE, Bradley JM, Hewitt $\mathrm{O}$, et al: Airway clearance in bronchiectasis: a randomized crossover trial of active cycle of breathing techniques versus Acapella®. Respiration 2005;72:239-42

15. Alves Silva CE, Santos JG, Jansen JM, et al: Laboratory evaluation of the Acapella device: pressure characteristics under different conditions, and a software tool to optimize its practical use. Respir Care 2009;54:1480-7 